Supplement of Hydrol. Earth Syst. Sci., 25, 3053-3070, 2021

https://doi.org/10.5194/hess-25-3053-2021-supplement

(c) Author(s) 2021. CC BY 4.0 License.

(c) (i)

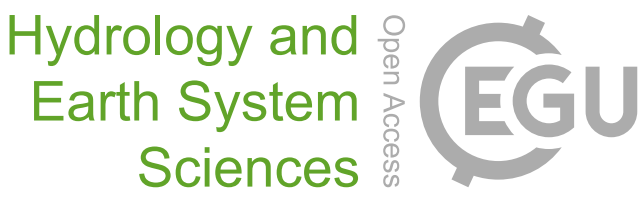

Supplement of

\title{
Groundwater fauna in an urban area - natural or affected?
}

Fabien Koch et al.

Correspondence to: Fabien Koch (fabien.koch@kit.edu)

The copyright of individual parts of the supplement might differ from the article licence. 

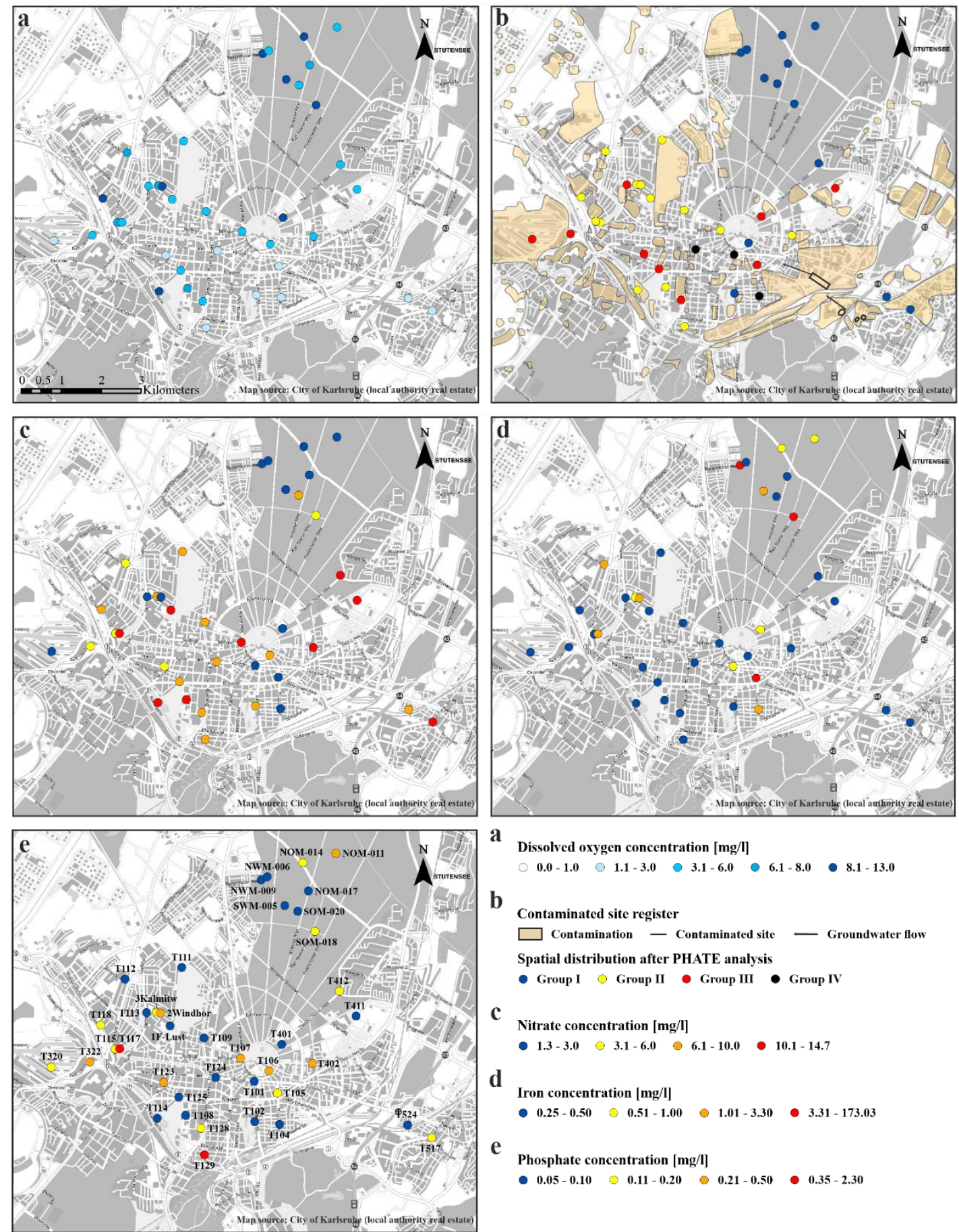

a Dissolved oxygen concentration [mg/l]

$$
0.0 \text { - } 1.0 \bigcirc 1.1 \text { - } 3.0 \quad 3.1-6.0 \quad 6.1 \text { - } 8.0 \bigcirc 8.1 \text { - } 13.0
$$

b

Contaminated site register

$\square$ Contamination - Contaminated site - Groundwater flow

Spatial distribution after PHATE analysis

- Group I $\bigcirc$ Group II - Group III • Group IV

C Nitrate concentration [mg/l]

- $1.3-3.0 \bigcirc 3.1-6.0 \bigcirc 6.1-10.0 \bigcirc 10.1-14.7$

d

Iron concentration $[\mathrm{mg} / \mathrm{l}]$

$0.25-0.50 \bigcirc 0.51-1.00 \bigcirc 1.01-3.30 \bigcirc 3.31-173.03$

e

Phosphate concentration [mg/l]

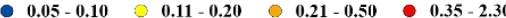

Figure S1: Overview map of Karlsruhe: (a) the average content of dissolved oxygen of the multiple measurements [mg/l]; (b) contaminated sites of the soil protection and contaminated site register (Bodenschutz- und Altlastenkataster) of Karlsruhe (modified after Stadt Karlsruhe, 2006; Kühlers et al., 2012; Wickert et al., 2006) and the spatial distribution after the PHATE analysis; (c) 5 average nitrate concentration [mg/l] of the repeated measurements; (d) iron concentration [mg/l] of the repeated measurements and (e) the phosphate concentration $[\mathrm{mg} / \mathrm{l}]$ of the repeated measurements at the bottom of the measurement wells. 


\section{Groundwater Fauna Index (GFI)}

The Groundwater-Fauna-Index (GFI), introduced by Hahn (2006), quantifies the ecological relevant conditions in the groundwater as a result of hydrological exchange between surface and groundwater. It incorporates ecologically important

10 groundwater parameters such as relative amount of detritus, variation of groundwater temperature and concentration of dissolved oxygen (Hahn, 2006) and is calculated by using Equation 1:

GW Fauna Index $=\sqrt{\text { Dissolved Oxygen }\left(\frac{m g}{l}\right)} \times \sqrt{\text { Relative Amount of Detritus }} \times$ Standard deviation Temperature (1)

The determined average GFI of all sampled wells is $6.0 \pm 2.8$ with a total variation between 0 and 14 and a heterogeneous distribution of the GFI-values. High GFI values (> 10, Type III), indicating hydrological exchange with the surface (Hahn,

15 2006), were only found in three wells which share a high standard deviation of GWT ( 2.6 to $3.5^{\circ} \mathrm{C}$ ), higher dissolved oxygen ( 5.5 to $5.8 \mathrm{mg} / \mathrm{l})$ as well as nitrate concentrations ( $7.7 \mathrm{up}$ to $12 \mathrm{mg} / \mathrm{l})$. These specific well locations have mainly no or minor sealed surfaces. Overall, $82 \%$ of the measurement wells showed meso-alimonic conditions (GFI > 2-10, Type II) and therefore indicate a medium level of surface influence, at diverse urban and forested locations. Only four wells in this study were well insulated from surface influences (GFI $<2$ ), with three wells located in densely built-up surroundings with sealed surfaces.

20 Moreover, the average GFI in the forested area is $4.5 \pm 1.9$ and in the urban area $6.2 \pm 2.7$.

\section{Shannon diversity index}

The Shannon-Index, introduced by Shannon and Weaver (1949) is an established standard method to quantify the ecological diversity of e.g. bacterial or faunistic communities. The index describes the diversity by including the number of species and the relative frequency of individuals. The sampled wells in the forested area show the highest balance (median $\mathrm{EH}=0.47)$ and

25 Shannon diversity index (median HS $=0.74$ ). The maximum diversity (median $\mathrm{H}_{\max }=1.58$ ) is the same in both the forested and the urban area. The balance (median EH $=0.42$ ) and Shannon diversity index (median HS $=0.52$ ) are only a little bit lower in the urban area. These results are comparable with the study of Brielmann et al. (2009), where the Shannon diversity index of an anthropogenically influenced groundwater of an aquifer downstream of an industrial facility varies between 0.20 and 1.45. Nevertheless, no clear distribution pattern according to faunal diversity is recognizable. Thus, the Shannon diversity 30 index was not considered further. 


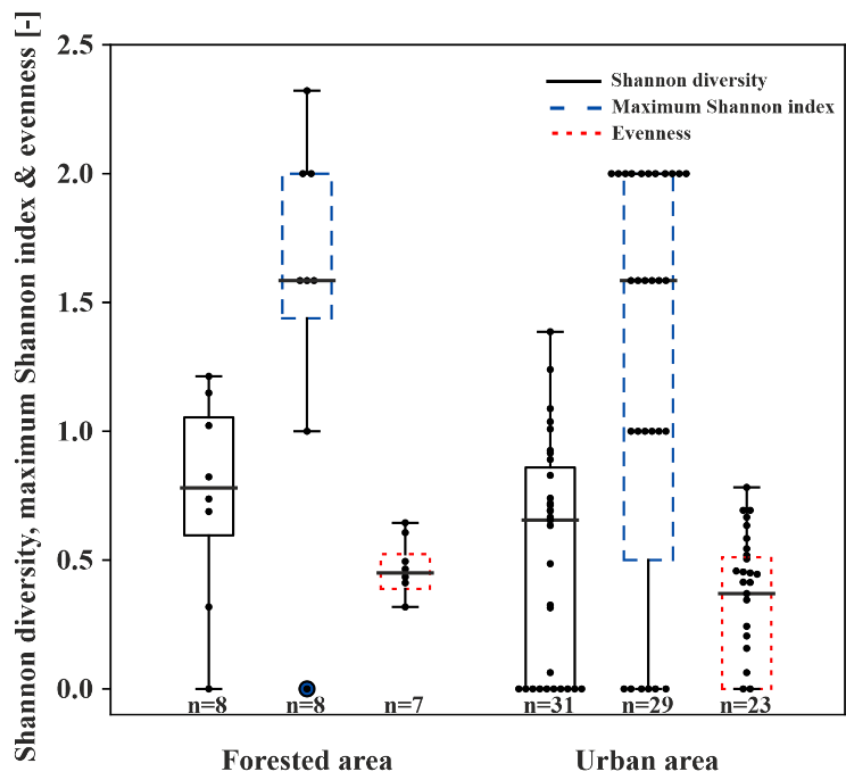

Figure S2. Boxplots of the Shannon diversity index, maximum Shannon index and evenness, divided in forested and urban area ( $n$ = number of wells, or number of wells at which the evaluation is applicable).

\section{Urban impacts on groundwater quality}

Urban impacts on groundwater systems can be manifold, such as increasing temperatures (urban heat islands (Menberg et al., 2013)), contaminants (Kuroda and Fukushi, 2008), changes in the precipitation discharge due to sealing, falling water levels due to groundwater withdrawal (Foster, 1990). In our study we intend to provide a first impression of the situation in Karlsruhe and therefore focus on the standard parameters. A first overview is given by the LUBW continuous monitoring program of groundwater wells (Landesanstalt für Umwelt Messungen und Naturschutz Baden-Württemberg, 2020), which provides profound groundwater analysis in Karlsruhe. Some of the considered measurement wells are close to the measurement wells of this study. Assessing the evaluation period of this study (2011-2014), most of the wells of the monitoring program show values within the range of the local background or below the thresholds of the drinking water ordinance of Germany and therefore no contamination.

45 One exception is a measurement well in the Kapellen-Street (next to T105), which shows higher ammonium (average: $0.55 \mathrm{mg} / \mathrm{l}$, threshold drinking water ordinance: $0.5 \mathrm{mg} / \mathrm{l})$, iron $(5.1 \mathrm{mg} / \mathrm{l}$, threshold value of the German drinking water ordinance: $0.2 \mathrm{mg} / \mathrm{l})$ and manganese concentrations $(0.55 \mathrm{mg} / \mathrm{l}$, threshold drinking water ordinance: $0.05 \mathrm{mg} / \mathrm{l})$. Moreover, this well has a noticeable concentration of arsenic $(8.7 \mu \mathrm{g} / \mathrm{l}$, threshold drinking water ordinance: $10 \mu \mathrm{g} / \mathrm{l})$ and of the herbizide CGA $369873(0.1 \mu \mathrm{g} / \mathrm{l}$, threshold: $0.1 \mu \mathrm{g} / \mathrm{l})$. This well is at the margin of one of the largest contaminated sites in Karlsruhe, the

50 former gas plant. 
Three other wells, which contain contaminants are in the Kaiserallee, Mathy-Street (next to T124) and near the municipal hospital. They showed noticeable concentrations of volatile hydrocarbons of up to $13 \mu \mathrm{g} / \mathrm{l}$ during the evaluation period (in detail at the hospital: 3-6 $\mu \mathrm{g} / \mathrm{l}$; Kaiserallee: 5-8 $\mu \mathrm{g} / \mathrm{l}$; Mathy Street: about $3 \mu \mathrm{g} / \mathrm{l})$. In comparison, the German threshold value of the drinking water ordinance is $20 \mu \mathrm{g} / \mathrm{l}$.

55 The groundwater of one measurement well in the Hardtwald (next to SWM-005/SOM-020) has a different chemical composition than the wells in the urban area. It shows lower concentrations of boron (30-45 $\mu \mathrm{g} / \mathrm{l}$, compared to the other wells: $50-98 \mu \mathrm{g} / \mathrm{l})$, calcium (100-110 mg/l, compared to the other wells of up to $150 \mathrm{mg} / \mathrm{l})$, chloride $(25.5 \mathrm{mg} / \mathrm{l}$ in 2014 compared to the other wells: > $50 \mathrm{mg} / \mathrm{l})$, potassium $(3.2 \mathrm{mg} / \mathrm{l})$ and sodium $(11.3 \mathrm{mg} / \mathrm{l})$. Furthermore, the content of dissolved oxygen is higher than in the wells of the urban area (average with $4.8 \mathrm{mg} / \mathrm{l}$ ).

60 This overview indicates that beside one larger and two smaller contaminations, the groundwater beneath Karlsruhe contains only minor pollution. Groundwater fauna can usually cope well with short-term changes of chemical-physical parameters (Griebler et al., 2016). Previous studies showed that some species can even benefit from pollutants (Matzke, 2006; Zuurbier et al., 2013). Thus, the main documented impacts on groundwater quality in the study area are related to groundwater temperature, oxygen and nitrate concentration.

65

Table S1: Estimation of the relative amounts of sediment per sample (modified after Hahn, 2006).

\begin{tabular}{lll}
\hline Scale & Description & Characterisation \\
\hline 0 & Absent & No sediments in the sampling vessel \\
1 & Little & Bottom of the sampling vessel $(\varnothing 1 / 47.6 \mathrm{~cm})$ slightly covered by sediment \\
2 & Much & Bottom of the sampling vessel covered by several millimetres of sediment \\
3 & Very much & Bottom of the sampling vessel covered by one or more centimetres of sediment \\
\hline
\end{tabular}


Table S2: Well locations, information, sampled properties and result of the evaluation (GWT=groundwater temperature, *sampling 2011-2012: 6 times, $\dagger$ sampling 2014: 3 times).

\begin{tabular}{|c|c|c|c|c|c|c|c|c|c|c|c|c|c|c|}
\hline $\begin{array}{c}\text { Measuring } \\
\text { point }\end{array}$ & Location & & $\begin{array}{c}\text { Area } \\
\text { classification }\end{array}$ & $\begin{array}{c}\text { Depth } \\
\text { [m] }\end{array}$ & $\begin{array}{c}\text { Average } \\
\text { GWT }\left[{ }^{\circ} \mathbf{C}\right]\end{array}$ & $\begin{array}{c}\text { SD GWT } \\
{[-]}\end{array}$ & $\begin{array}{c}\text { Relative } \\
\text { amount of } \\
\text { detritus [-] }\end{array}$ & $\begin{array}{c}\text { Average } \\
\text { dissolved } \\
\text { oxygen }[\mathrm{mg} / \mathrm{l}]\end{array}$ & $\begin{array}{c}\text { Average } \\
\text { GFI [-] }\end{array}$ & $\begin{array}{c}\text { Amount } \\
\text { crustaceans } \\
\quad(\text { acc. to } \\
\text { Griebler et } \\
\text { al. (2014)) } \\
{[\%]}\end{array}$ & $\begin{array}{c}\text { Amount } \\
\text { oligochaetes } \\
\text { (acc. to } \\
\text { Griebler et al. } \\
\text { (2014)) [\%] }\end{array}$ & $\begin{array}{c}\text { Total } \\
\text { amount of } \\
\text { individuals } \\
{[-]}\end{array}$ & $\begin{array}{l}\text { Numbers } \\
\text { of taxa [-] }\end{array}$ & $\begin{array}{c}\text { Ecological } \\
\text { condition (acc. to } \\
\text { Griebler et al. } \\
(\mathbf{2 0 1 4 )})\end{array}$ \\
\hline T101 & Lammstr. No.7 & $*$ & Urban area & 39.0 & 14.4 & 0.05 & 3 & 0.97 & 0 & 0 & 0 & 0 & 0 & $\begin{array}{c}\text { Faunistic evaluation } \\
\text { not possible }\end{array}$ \\
\hline T102 & Tulla Bad & $*$ & Urban area & 10.0 & 14.0 & 2.99 & 2 & 1.45 & 5 & 100 & 0 & 4 & 2 & Natural \\
\hline T104 & Arbeitsamt - Rankestr. & $*$ & Urban area & 15.8 & 12.5 & 1.28 & 3 & 1.07 & 2 & 0 & 0 & 0 & 0 & $\begin{array}{c}\text { Faunistic evaluation } \\
\text { not possible }\end{array}$ \\
\hline T105 & Fritz-Erler-Str. No.21 & $*$ & Urban area & 9.3 & 14.4 & 3.29 & 3 & 1.29 & 6 & 0 & 100 & 1 & 1 & $\begin{array}{c}\text { Faunistic evaluation } \\
\text { not possible }\end{array}$ \\
\hline T106 & Schloßplatz / Schloßbezirk & $*$ & Urban area & 11.0 & 14.2 & 2.60 & 3 & 4.02 & 9 & 86 & 14 & 7 & 3 & Natural \\
\hline $\mathrm{T} 123$ & Sophienstr. - Grillparzerstr. & $*$ & Urban area & 14.0 & 12.8 & 1.65 & 1 & 2.18 & 2 & 0 & 100 & 3 & 2 & Affected \\
\hline $\mathrm{T} 124$ & Kaiserplatz & $*$ & Urban area & 13.0 & 14.9 & 1.95 & 3 & 1.97 & 5 & 0 & 0 & 2 & 1 & Affected \\
\hline $\mathrm{T} 125$ & Kriegsstr. No.141 & $*$ & Urban area & 11.8 & 15.0 & 2.32 & 1 & 3.64 & 4 & 0 & 100 & 103 & 3 & Affected \\
\hline $\mathrm{T} 128$ & Südendstr. - Brauerstr. & $*$ & Urban area & 9.5 & 13.2 & 3.21 & 2 & 5.50 & 11 & 0 & 100 & 13 & 2 & Affected \\
\hline T129 & Schule Beiertheim & $*$ & Urban area & 8.5 & 12.2 & 3.67 & 1 & 2.31 & 6 & 91 & 9 & 124 & 4 & Natural \\
\hline $\mathrm{T} 320$ & Südbeckenstr. No.16 & $*$ & Urban area & 9.0 & 12.6 & 3.43 & 1 & 2.62 & 6 & 0 & 100 & 252 & 1 & Affected \\
\hline T322 & Rheinhafenbad & $*$ & Urban area & 10.0 & 15.9 & 2.99 & 2 & 3.45 & 8 & 0 & 100 & 6 & 2 & Affected \\
\hline $\mathrm{T} 402$ & Am Fasanengarten - Parkstr. & $*$ & Urban area & 9.0 & 12.4 & 3.43 & 2 & 3.68 & 9 & 50 & 50 & 4 & 4 & Affected \\
\hline $\mathrm{T} 411$ & Gewann Blösse & $*$ & Urban area & 10.9 & 11.3 & 2.64 & 3 & 5.74 & 11 & 0 & 100 & 34 & 2 & Affected \\
\hline $\mathrm{T} 412$ & Theodor- Heuss - Allee & $*$ & Urban area & 10.0 & 11.4 & 2.99 & 1 & 4.47 & 6 & 100 & 0 & 6 & 4 & Natural \\
\hline T517 & Auer Str. - Reichenbachstr. & $*$ & Urban area & 9.0 & 12.8 & 3.43 & 2 & 2.84 & 8 & 100 & 0 & 5 & 1 & Natural \\
\hline T524 & Dornwaldstr. & $*$ & Urban area & 9.0 & 11.5 & 3.43 & 2 & 1.77 & 6 & 99 & 1 & 275 & 2 & Natural \\
\hline T401 & $\begin{array}{l}\text { Area next to the Wildpark- } \\
\text { Stadion }\end{array}$ & $\dagger$ & Urban area & 11.0 & 14.2 & 2.60 & 2 & 8.90 & 10 & 0 & 100 & 8 & 1 & Affected \\
\hline T109 & Erzbergerstr. & $\dagger$ & Urban area & 13.7 & 14.1 & 1.76 & 1 & 4.86 & 4 & 92 & 8 & 38 & 4 & Natural \\
\hline T108 & $\begin{array}{l}\text { Edgar-von-Gierke-Str. - } \\
\text { Siegfried-Kühn-Str. }\end{array}$ & $\dagger$ & Urban area & 12.0 & 14.9 & 2.26 & 2 & 6.12 & 8 & 79 & 21 & 25 & 4 & Affected \\
\hline T114 & Allotment garden at the Alb & $\dagger$ & Urban area & 12.8 & 15.4 & 2.01 & 1 & 8.25 & 6 & 13 & 88 & 171 & 4 & Affected \\
\hline
\end{tabular}




\begin{tabular}{|c|c|c|c|c|c|c|c|c|c|c|c|c|c|}
\hline $\mathrm{T} 115$ & Sonnenstr. - Zietenstr. & $\dagger$ Urban area & 13.5 & 14.8 & 1.81 & 1 & 4.83 & 4 & 89 & 11 & 23 & 4 & Natural \\
\hline $\mathrm{T} 117$ & Sonnenstr. - Zietenstr. & $\dagger$ Urban area & 13.0 & 17.0 & 1.95 & 3 & 6.24 & 8 & 92 & 8 & 76 & 5 & Natural \\
\hline $\mathrm{T} 118$ & Schoemperlenstr. & $\dagger$ Urban area & 13.7 & 16.1 & 1.76 & 1 & 8.45 & 6 & 38 & 63 & 11 & 4 & Affected \\
\hline $\mathrm{T} 112$ & Wattstr. - Annweilerstr. & $\dagger$ Urban area & 12.0 & 14.5 & 2.26 & 2 & 6.87 & 8 & 100 & 0 & 204 & 4 & Natural \\
\hline $\mathrm{T} 111$ & Field near Kaiserslauterner-Str. & $\dagger$ Urban area & 8.9 & 13.4 & 3.48 & 3 & 5.75 & 14 & 77 & 23 & 96 & 4 & Affected \\
\hline $\mathrm{T} 113$ & Hertzstr. - St. Barbara-Weg & $\dagger$ Urban area & 11.0 & 17.5 & 2.60 & 1 & 3.35 & 5 & 0 & 100 & 1 & 1 & Affected \\
\hline 3Kalmitw & Kalmitweg No.3 & $\dagger$ Urban area & 15.5 & 15.3 & 1.34 & 1 & 6.44 & 3 & 77 & 23 & 13 & 3 & Affected \\
\hline 2Windhor & $\begin{array}{l}\text { Wilhelm-Windhorststr. - } \\
\text { Schänzle }\end{array}$ & $\dagger$ Urban area & 15.2 & 15.8 & 1.34 & 2 & 8.64 & 6 & 20 & 80 & 353 & 4 & Affected \\
\hline 1F-Lust- & Franz-Lust-Str. - Kußmaulstr. & $\dagger$ Urban area & 15.2 & 17.3 & 1.34 & 2 & 5.95 & 5 & 65 & 35 & 630 & 3 & Affected \\
\hline $\mathrm{T} 107$ & Molkestr. - Willy-Brandt-Allee & $\dagger$ Urban area & 10.1 & 16.2 & 2.95 & 1 & 4.90 & 7 & 66 & 34 & 130 & 3 & Affected \\
\hline NOM-011 & & $\dagger$ Forested area & 14.9 & 10.7 & 1.47 & 1 & 3.42 & 3 & 100 & 0 & 15 & 1 & Natural \\
\hline NOM-017 & & $\dagger$ Forested area & 15.0 & 10.9 & 1.45 & 3 & 7.20 & 7 & 97 & 3 & 506 & 6 & Natural \\
\hline SOM-020 & & $\dagger$ Forested area & 15.0 & 10.7 & 1.45 & 1 & 5.81 & 3 & 50 & 50 & 9 & 4 & Affected \\
\hline SOM-018 & & $\dagger$ Forested area & 27.0 & 10.3 & 0.26 & 3 & 12.75 & 2 & 90 & 10 & 31 & 2 & Natural \\
\hline SWM-005 & Hardtwald & $\dagger$ Forested area & 15.5 & 10.5 & 1.34 & 2 & 8.72 & 6 & 26 & 74 & 358 & 3 & Affected \\
\hline NWM-009 & & $\dagger$ Forested area & 15.0 & 10.8 & 1.45 & 2 & 10.69 & 7 & 43 & 57 & 90 & 4 & Affected \\
\hline NWM-006 & & $\dagger$ Forested area & 14.8 & 10.7 & 1.49 & 1 & 5.00 & 3 & 86 & 14 & 16 & 3 & Natural \\
\hline NOM-014 & & $\dagger$ Forested area & 15.0 & 10.5 & 1.45 & 1 & 9.92 & 5 & 67 & 33 & 23 & 3 & Affected \\
\hline
\end{tabular}


Table S3: Taxa-site matrix of the invertebrate fauna of each water gauge.

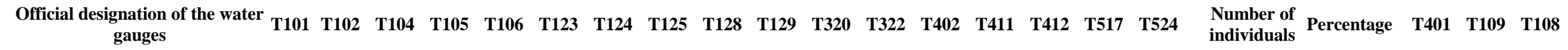

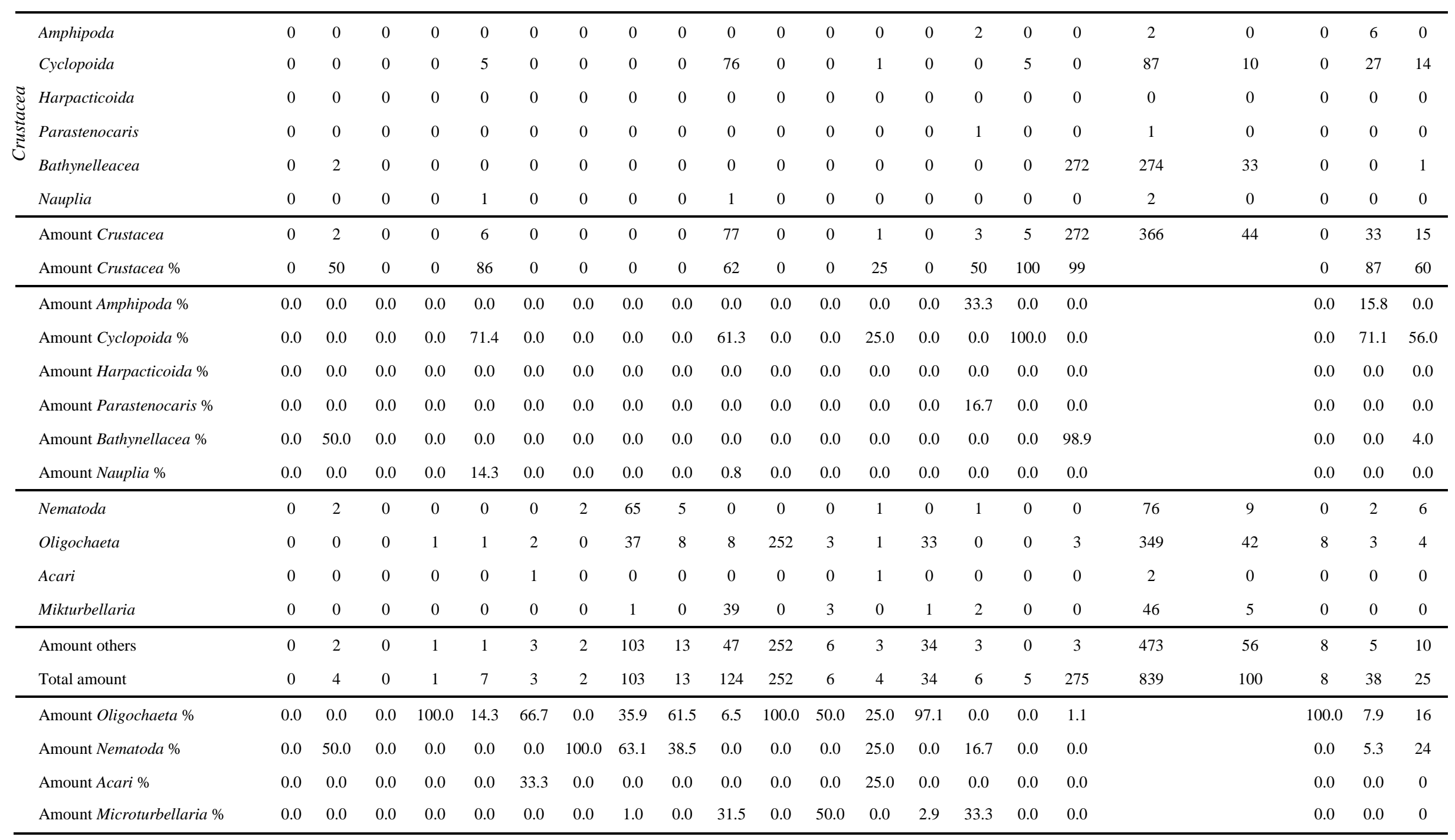




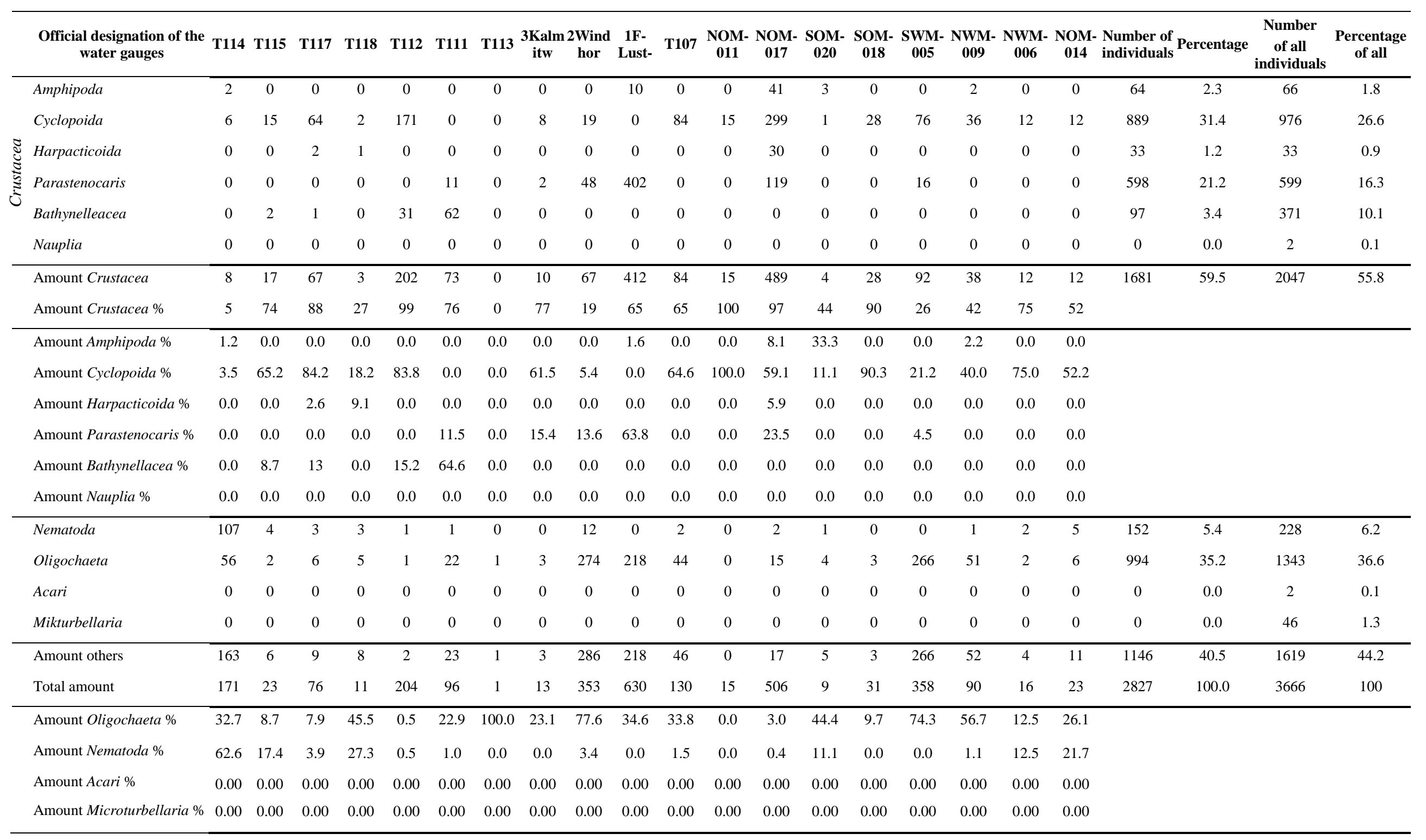


Table S4: Average and standard deviation of faunistic, chemical and physical parameters with regard to the four groups (result of the PHATE analysis).

\begin{tabular}{|c|c|c|c|c|c|c|c|c|c|}
\hline & $\begin{array}{c}\text { Average amount } \\
\text { crustaceans (acc. to } \\
\text { Griebler et al. } \\
(2014))[\%]\end{array}$ & $\begin{array}{l}\text { Average amount } \\
\text { oligochaetes (acc. to } \\
\text { Griebler et al. } \\
(\mathbf{2 0 1 4}))[\%]\end{array}$ & $\begin{array}{c}\text { Average } \\
\text { numbers of } \\
\text { taxa }[-]\end{array}$ & $\begin{array}{l}\text { Average total } \\
\text { amount of } \\
\text { individuals [-] }\end{array}$ & $\begin{array}{c}\text { Average } \\
\text { Shannon } \\
\text { diversity [-] }\end{array}$ & $\begin{array}{c}\text { Average } \\
\text { abundance } \\
\text { Amphipoda [-] }\end{array}$ & $\begin{array}{c}\text { Average } \\
\text { abundance } \\
\text { Cyclopoida [-] }\end{array}$ & $\begin{array}{c}\text { Average } \\
\text { abundance } \\
\text { Parastenocaris [-] }\end{array}$ & $\begin{array}{c}\text { Average } \\
\text { abundance } \\
\text { Bathynellacea [-] }\end{array}$ \\
\hline Group I $(n=13)$ & $80.3( \pm 24.5)$ & $19.7( \pm 24.5)$ & $2.9( \pm 1.3)$ & $103.5( \pm 159.6)$ & $0.6( \pm 0.4)$ & $3.7( \pm 10.8)$ & $37.6( \pm 78.2)$ & $10.5( \pm 31.6)$ & $21.1( \pm 72.4)$ \\
\hline Group II (n = 14) & $67.8( \pm 26.7)$ & $32.4( \pm 26.9)$ & $3.9( \pm 0.5)$ & $135.6( \pm 165.9)$ & $0.9( \pm 0.3)$ & $1.3( \pm 2.9)$ & $34.8( \pm 46.9)$ & $33.1( \pm 103.1)$ & $6.9( \pm 17.2)$ \\
\hline Group III (n = 9) & $0.0( \pm 0.0)$ & $100.0( \pm 0.0)$ & $1.7( \pm 0.7)$ & $46.8( \pm 78.8)$ & $0.2( \pm 0.3)$ & $0.0( \pm 0.0)$ & $0.0( \pm 0.0)$ & $0.0( \pm 0.0)$ & $0.0( \pm 0.0)$ \\
\hline Group IV(n = 3) & $0.0( \pm 0.0)$ & $0.0( \pm 0.0)$ & $0.3( \pm 0.5)$ & $0.7( \pm 0.9)$ & $0.0( \pm 0.0)$ & $0.0( \pm 0.0)$ & $0.0( \pm 0.0)$ & $0.0( \pm 0.0)$ & $0.0( \pm 0.0)$ \\
\hline
\end{tabular}

\begin{tabular}{lcccccc}
\hline & $\begin{array}{c}\text { Average } \\
\text { geological unit [-] }\end{array}$ & $\begin{array}{c}\text { Average GWT } \\
{\left[{ }^{\circ} \mathbf{C}\right]}\end{array}$ & $\begin{array}{c}\text { Average phosphate } \\
\text { concentration[mg/l] }\end{array}$ & $\begin{array}{c}\text { Average nitrate } \\
\text { concentration [mg/I] }\end{array}$ & $\begin{array}{c}\text { Average relative } \\
\text { amount of detritus [-] }\end{array}$ & $\begin{array}{c}\text { Average } \\
\text { depth [m] }\end{array}$ \\
\hline Group I (n= 13) & $2( \pm 1)$ & $11.5( \pm 1.3)$ & $0.1( \pm 0.1)$ & $5.4( \pm 3.9)$ & $1.8( \pm 0.8)$ & $13.9( \pm 4.5)$ \\
Group II (n= 14) & $3( \pm 1)$ & $15.0( \pm 1.5)$ & $0.3( \pm 0.6)$ & $9.1( \pm 3.7)$ & $1.7( \pm 0.7)$ & $12.4( \pm 2.3)$ \\
Group III (n= 9) & $3( \pm 1)$ & $14.1( \pm 1.8)$ & $0.1( \pm 0.1)$ & $4.9( \pm 3.9)$ & $1.8( \pm 0.8)$ & $10.7( \pm 1.5)$ \\
Group IV(n= 3) & $2( \pm 1)$ & $13.9( \pm 1.0)$ & $0.1( \pm 0.0)$ & $3.9( \pm 3.4)$ & $3.0( \pm 0.0)$ & $22.6( \pm 11.7)$ \\
\hline
\end{tabular}


Table S5: Results of the Mann-Whitney-Tests from the four groups of the PHATE analysis.

\begin{tabular}{|c|c|c|c|c|c|c|c|c|c|c|}
\hline & $\begin{array}{l}\text { Amount crustaceans } \\
\text { (acc. to Griebler et } \\
\text { al. (2014)) [\%] }\end{array}$ & \multicolumn{2}{|c|}{$\begin{array}{l}\text { Amount oligochaetes } \\
\text { (acc. to Griebler et } \\
\text { al. }(2014))[\%]\end{array}$} & $\begin{array}{l}\text { Numbers } \\
\text { of taxa [-] }\end{array}$ & $\begin{array}{c}\text { Total amount } \\
\text { of individuals } \\
{[-]}\end{array}$ & $\begin{array}{cc}\text { Shannon } & A \\
\text { diversity } & A \\
{[-]} & \end{array}$ & $\begin{array}{l}\text { Abundance } \\
\text { Amphipoda } \\
\text { [-] }\end{array}$ & $\begin{array}{c}\text { Abundance } \\
\text { Cyclopoida } \\
{[-]}\end{array}$ & $\begin{array}{c}\text { Abundance } \\
\text { Parastenocaris } \\
{[-]}\end{array}$ & $\begin{array}{c}\text { Abundance } \\
\text { Bathynellacea } \\
{[-]}\end{array}$ \\
\hline Group I vs. II (n = 13;14) & $1.3 \times 10^{-1}$ & \multicolumn{2}{|c|}{$1.3 \times 10^{-1}$} & $1.5 \times 10^{-2}$ & $2.0 \times 10^{-1}$ & $3.2 \times 10^{-1}$ & $7.4 \times 10^{-1}$ & $5.6 \times 10^{-1}$ & $7.2 \times 10^{-1}$ & $4.0 \times 10^{-1}$ \\
\hline Group I vs. III (n = 13;9) & $4.0 \times 10^{-6}$ & \multicolumn{2}{|c|}{$4.0 \times 10^{-6}$} & $2.7 \times 10^{-2}$ & $2.4 \times 10^{-1}$ & $3.9 \times 10^{-1}$ & $2.0 \times 10^{-1}$ & $8.9 \times 10^{-4}$ & $3.7 \times 10^{-1}$ & $6.8 \times 10^{-1}$ \\
\hline Group IV vs. I $(n=3 ; 13)$ & $3.6 \times 10^{-3}$ & \multicolumn{2}{|c|}{$1.3 \times 10^{-1}$} & $1.1 \times 10^{-2}$ & $3.6 \times 10^{-3}$ & $2.0 \times 10^{-1}$ & $7.9 \times 10^{-1}$ & $7.1 \times 10^{-2}$ & 1.0 & 1.0 \\
\hline Group II vs. III (n = 14;9) & $2.5 \times 10^{-6}$ & \multicolumn{2}{|c|}{$2.5 \times 10^{-6}$} & $9.8 \times 10^{-7}$ & $3.4 \times 10^{-2}$ & $3.3 \times 10^{-2}$ & $4.1 \times 10^{-1}$ & $1.4 \times 10^{-4}$ & $2.3 \times 10^{-1}$ & $1.2 \times 10^{-1}$ \\
\hline Group IV vs. II (n = 3;14) & $2.9 \times 10^{-3}$ & \multicolumn{2}{|c|}{$1.2 \times 10^{-2}$} & $2.9 \times 10^{-3}$ & $2.9 \times 10^{-3}$ & $2.9 \times 10^{-2}$ & 1.0 & $2.9 \times 10^{-2}$ & $8.4 \times 10^{-1}$ & $6.5 \times 10^{-1}$ \\
\hline \multirow[t]{2}{*}{ Group IV vs. III (n = 3;9) } & 1.0 & \multicolumn{2}{|c|}{$9.1 \times 10^{-3}$} & $4.6 \times 10^{-2}$ & $2.7 \times 10^{-2}$ & $7.6 \times 10^{-1}$ & 1.0 & 1.0 & 1.0 & 1.0 \\
\hline & Geological unit [-] & GWT $\left[{ }^{\circ} \mathbf{C}\right]$ & \multicolumn{2}{|c|}{$\begin{array}{c}\text { Phosphate } \\
\text { concentration } \\
{[\mathrm{mg} / \mathrm{l}]}\end{array}$} & $\begin{array}{c}\text { Nitrate } \\
\text { concentration } \\
{[\mathrm{mg} / \mathrm{l}]}\end{array}$ & $\begin{array}{c}\text { Relative } \\
\text { amount of } \\
\text { detritus [-] }\end{array}$ & \multicolumn{2}{|c|}{ Depth $[\mathrm{m}]$} & & \\
\hline Group I vs. II (n = 13;14) & $8.2 \times 10^{-3}$ & $2.0 \times 10^{-5}$ & $5.2 \times 1$ & & $1.2 \times 10^{-2}$ & $6.1 \times 10^{-1}$ & $2.8 \times 10$ & & & \\
\hline Group I vs. III $(\mathrm{n}=13 ; 9)$ & $1.5 \times 10^{-1}$ & $3.8 \times 10^{-3}$ & 1.0 & & $9.9 \times 10^{-1}$ & $9.7 \times 10^{-1}$ & $7.4 \times 10$ & & & \\
\hline Group IV vs. I (n = 3;13) & $4.8 \times 10^{-1}$ & $2.1 \times 10^{-2}$ & $3.6 \times 1$ & & $6.1 \times 10^{-1}$ & $7.1 \times 10^{-1}$ & $2.2 \times 10$ & & & \\
\hline Group II vs. III (n = 14;9) & $4.4 \times 10^{-1}$ & $3.2 \times 10^{-1}$ & $7.2 \times 1$ & & $4.5 \times 10^{-2}$ & $8.5 \times 10^{-1}$ & $9.4 \times 10$ & & & \\
\hline Group IV vs. II (n = 3;14) & $3.5 \times 10^{-1}$ & $2.8 \times 10^{-1}$ & $2.9 \times 1$ & & $9.1 \times 10^{-2}$ & $2.9 \times 10^{-2}$ & $1.1 \times 10$ & & & \\
\hline Group IV vs. III $(n=3 ; 9)$ & $8.4 \times 10^{-1}$ & $9.6 \times 10^{-1}$ & $9.1 \times 1$ & & $1.7 \times 10^{-1}$ & $9.1 \times 10^{-2}$ & $1.8 \times 10$ & & & \\
\hline
\end{tabular}



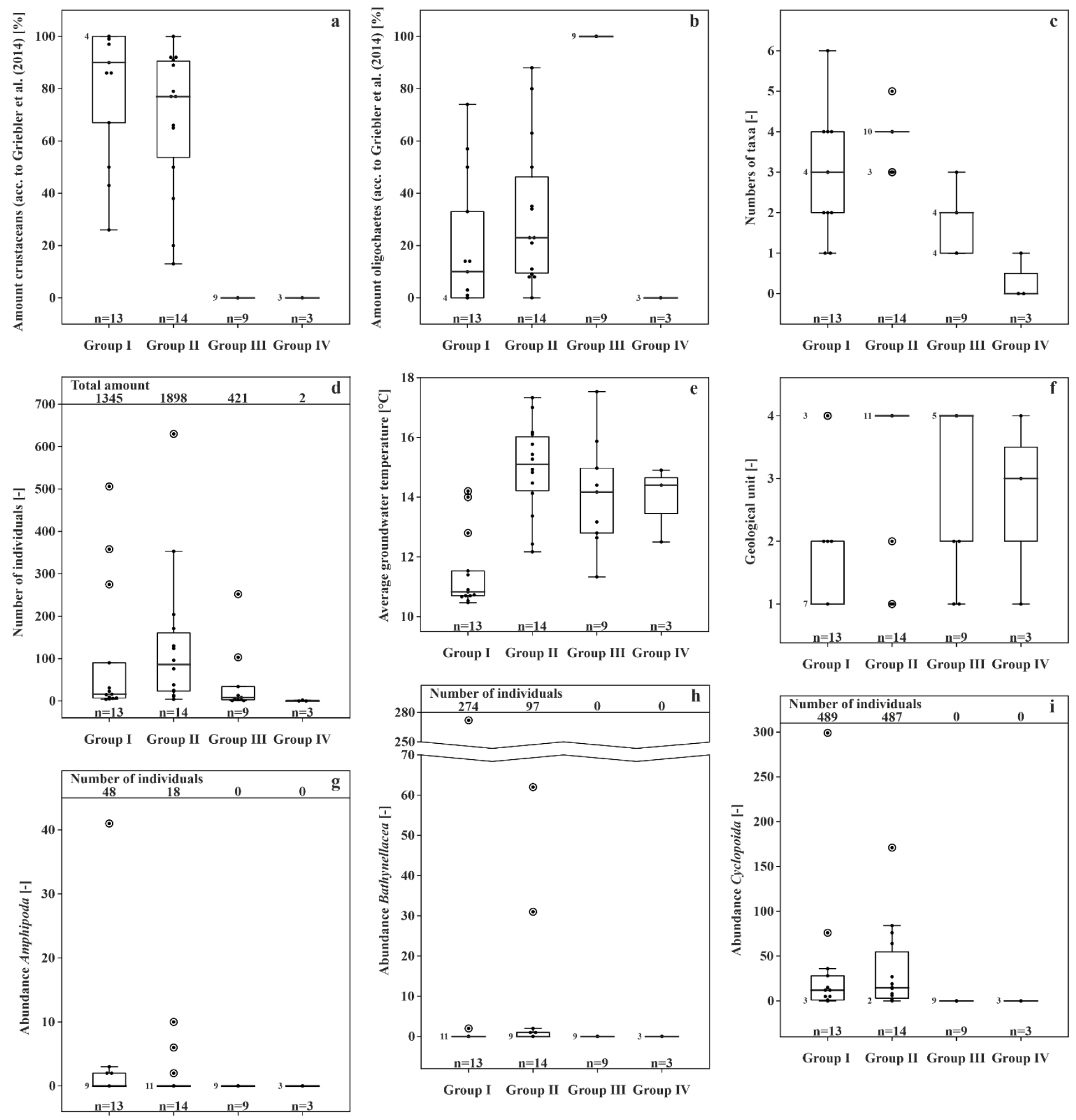

Group I Group II Group III Group IV

Figure S3. Boxplots of: (a) Amount of crustaceans [\%] and (b) oligochaetes [\%] according to the scheme of Griebler et al. (2014); (c) numbers of Taxa [-]; (d) number of individuals [-]; (e) average GWT of the repeated measurements at the bottom of the measurement wells [\%] and (f) geological unit [-]; (g) Abundance of the order Amphipoda [-]; (h) of the order Bathynellacea [-] and (i) of the order Cyclopoida [-], divided into four groups according to the PHATE visualization $(n=$ number of wells). 


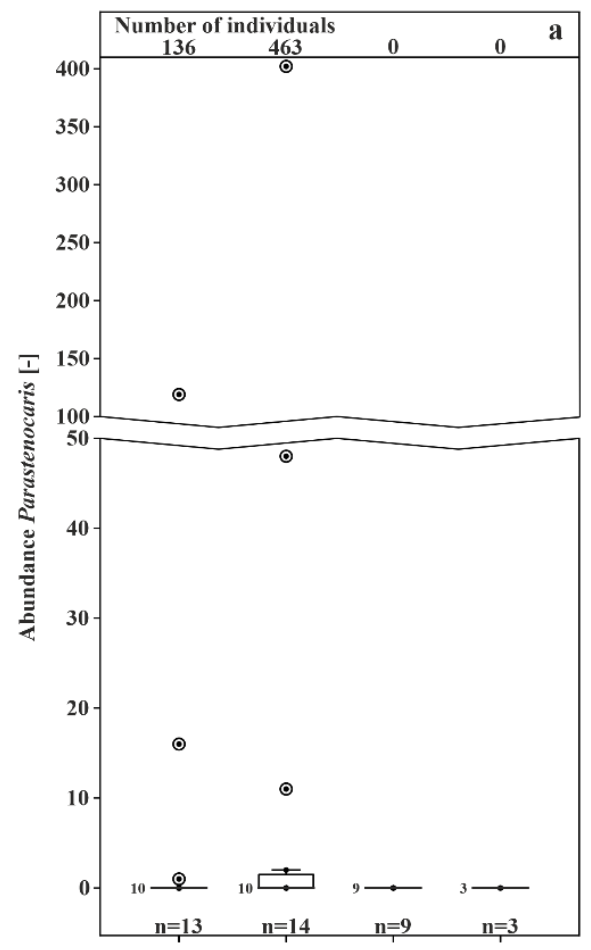

Group I Group II Group III Group IV

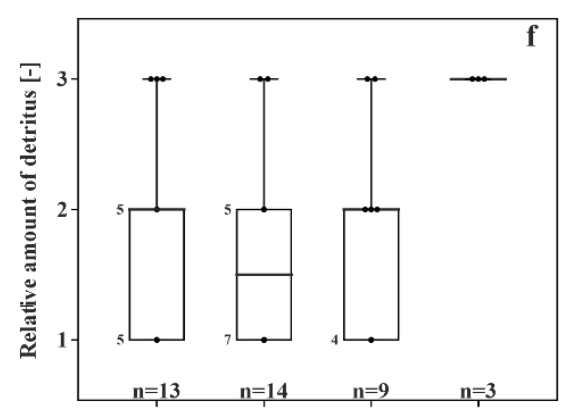

Group I Group II Group III Group IV
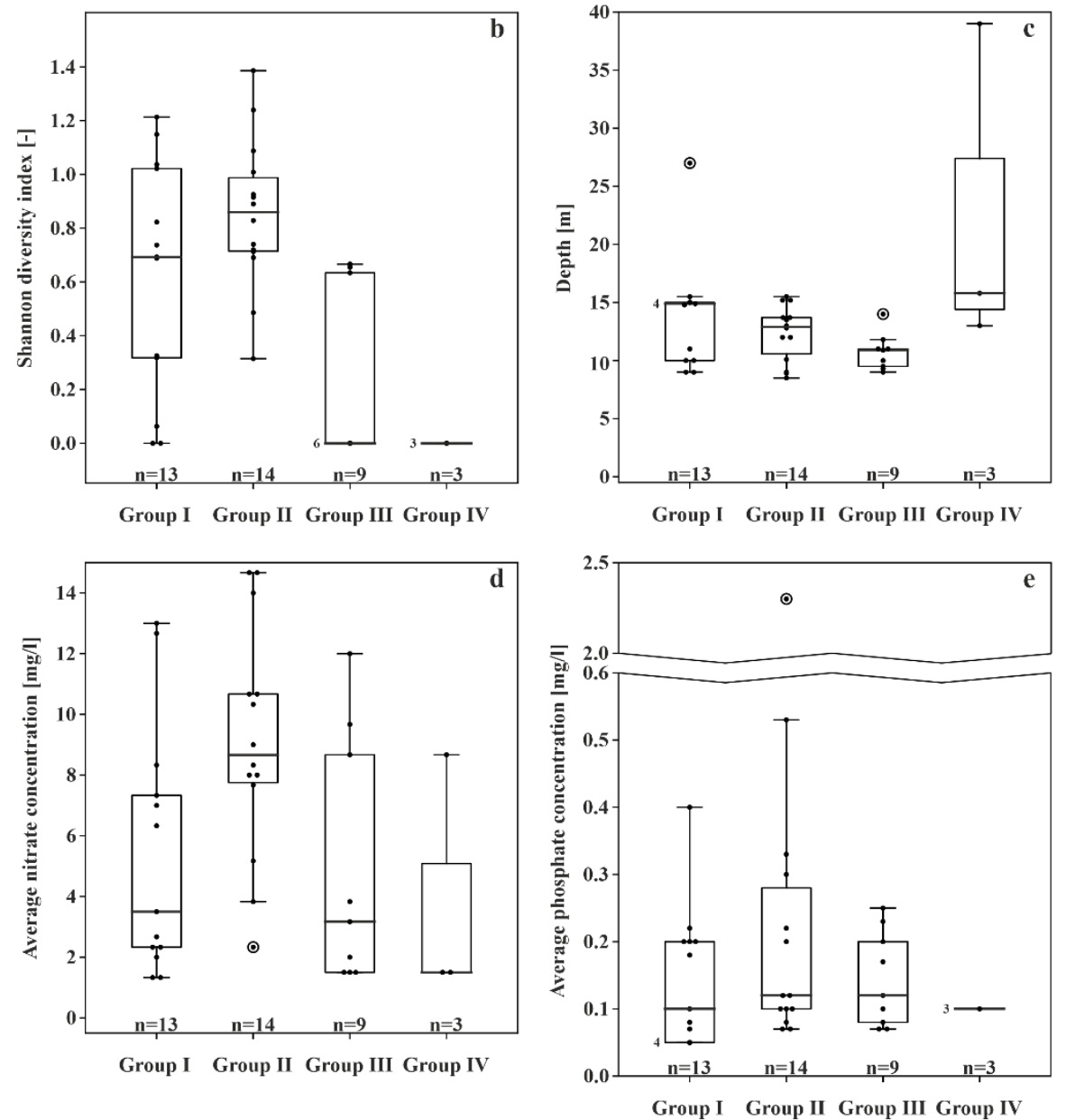

Figure S4. Boxplots of: (a) Abundance of the genus Parastenocaris [-]; (b) Shannon diversity index [-]; (c) depth of the measurement wells $[\mathrm{m}]$; (d) of the average nitrate concentration [mg/l]; (e) average phosphate concentration [mg/l] of the repeated measurements at the bottom of the measurement wells and (f) of the relative amount of detritus [-], divided into four groups according to the PHATE visualization $(n=$ number of wells $)$. 


\section{References}

Brielmann, H., Griebler, C., Schmidt, S. I., Michel, R. and Lueders, T.: Effects of thermal energy discharge on shallow groundwater ecosystems, FEMS Microbiol. Ecol., 68(3), 273-286, doi:10.1111/j.1574-6941.2009.00674.x, 2009.

Foster, S. S. D.: Impacts of urbanization on groundwater, Hydrol. Process. Water Manag. Urban Areas (Proceedings Duisb.

105 Symp. 1988), 198(198), 209-216 [online] Available from: https://www.researchgate.net/profile/Stephen_Foster11/publication/237480481_Impacts_of_urbanisation_on_groundwater/li nks/55b4d97508ae092e96557248/Impacts-of-urbanisation-on-groundwater.pdf, 1990.

Griebler, C., Brielmann, H., Haberer, C. M., Kaschuba, S., Kellermann, C., Stumpp, C., Hegler, F., Kuntz, D., WalkerHertkorn, S. and Lueders, T.: Potential impacts of geothermal energy use and storage of heat on groundwater quality,

110 biodiversity, and ecosystem processes, Environ. Earth Sci., 75(20), 1-18, doi:10.1007/s12665-016-6207-z, 2016.

Hahn, H. J.: A first approach to a quantitative ecological assessment of groundwater habitats: The GW-Fauna-Index, Limnologica, 36(2), 119-137, 2006.

Kühlers, D., Maier, M. and Roth, K.: Sanierung im Verborgenen, TerraTech Sanierungspraxis, 3, 14-16, 2012.

Kuroda, K. and Fukushi, T.: Groundwater Management in Asian Cities, Groundw. Manag. Asian Cities, (September 2014), 115 334, doi:10.1007/978-4-431-78399-2, 2008.

Landesanstalt für Umwelt Messungen und Naturschutz Baden-Württemberg: Jahresdatenkatalog Grundwasser, [online] Available from: http://jdkgw.lubw.baden-wuerttemberg.de/servlet/is/200/, 2020.

Matzke, D.: Untersuchungen zum Verhalten von Grundwasserfauna in Altlastflächen mit vorangegangenem Vergleich unterschiedlicher Sammeltechniken., 2006.

120 Menberg, K., Bayer, P., Zosseder, K., Rumohr, S. and Blum, P.: Subsurface urban heat islands in German cities, Sci. Total Environ., 442, 123-133, doi:10.1016/j.scitotenv.2012.10.043, 2013.

Shannon, C. E. and Weaver, W.: The mathematical theory of communication, The University of Illinois Press., 1949.

Stadt Karlsruhe: Bodenschutz- und Altlastenkataster der Stadt Karlsruhe, [online] Available from: https://www.karlsruhe.de/b3/natur_und_umwelt/umweltschutz/altlasten.de (Accessed 23 October 2019), 2006.

125 Wickert, F., Müller, A., Schäfer, W. and Tiehm, A.: Vergleich hochauflösender Grundwasserprobennahmeverfahren zur Charakterisierung der vertikalen LCKW-Verteilung im Grundwasserleiter, Altlastenspektrum, 01, 29-35, 2006.

Zuurbier, K. G., Hartog, N., Valstar, J., Post, V. E. A. and Van Breukelen, B. M.: The impact of low-temperature seasonal aquifer thermal energy storage (SATES) systems on chlorinated solvent contaminated groundwater: Modeling of spreading and degradation, J. Contam. Hydrol., 147, 1-13, doi:10.1016/j.jconhyd.2013.01.002, 2013. 
